\title{
Atlas-Guided Global Tractography: Imposing a Prior on the Local Track Orientation
}

\author{
Daan Christiaens, Marco Reisert, Thijs Dhollander, Frederik Maes, Stefan Sunaert, \\ and Paul Suetens
}

\begin{abstract}
Since its introduction over a decade ago, diffusion tractography has come a long way from local, deterministic methods, over probabilistic approaches, towards global tractography. Yet, the development of tractography methods has been largely focused on single subject data, and very little on cross-population analysis and intersubject variability. In this work, we extend global tractography with a prior on the local track orientation distribution (TOD), derived from 20 normal subjects. The proposed method is evaluated in 5 independent subjects. Results show that adding such prior regularizes the reconstructed track distribution, although registration errors can induce local artefacts. We conclude that atlas-guided global tractography can improve the fibre reconstruction and ultimately detect and quantify inter-subject differences in tractography.
\end{abstract}

\section{Introduction}

Since its introduction over a decade ago, diffusion tractography has come a long way from local, deterministic methods, over probabilistic approaches, towards global tractography $[10,12]$, steadily gaining importance for in vivo neuroanatomy studies and neurosurgical planning. Deterministic streamline approaches [14] are highly

D. Christiaens · T. Dhollander · F. Maes · P. Suetens

Medical Image Computing, Dept. of Electrical Engineering, KU Leuven, Belgium;

Medical Imaging Research Center, University Hospitals Leuven, Belgium;

iMinds, Medical IT Department, Belgium

e-mail: daan.christiaens@esat.kuleuven.be

M. Reisert

Medical Physics, Dept. of Radiology, University of Freiburg Medical Center, Germany

S. Sunaert

Translational MRI, Dept. of Imaging \& Pathology, KU Leuven, Belgium;

Medical Imaging Research Center, University Hospitals Leuven, Belgium 
sensitive to local estimation errors, leading to low accuracy, sensitivity and specificity of the reconstructed tracks in the presence of noise, modelling errors, and partial volume effects $[10,16]$. Probabilistic fibre tracking can accommodate for this uncertainty [16], but won't improve the quality of each individual streamline. Moreover, streamline tractography is inherently difficult to quantify due to its dependence on the seeding distribution. Global fibre tracking methods $[6,11,13,18]$ aim to reconstruct the ensemble of fibres that best explain the measured DWI data [12]. By addressing the problem at a global scale, these methods can be less sensitive to local estimation errors and maintain a quantifiable correspondence to the data.

Yet, the development of tractography methods has been largely focused on single subject data, and very little on cross-population analysis and inter-subject variability. Indeed, while atlases are at the core of state-of-the-art segmentation and label fusion methods [1], their use in diffusion tractography is mostly limited to automated and consistent delineation of regions of interest for seeding, inclusion, and exclusion in streamline tractography, and for clustering and labelling of the resulting fibre tracks [15]. In regard to streamline tractography, Cook et al. [3] have demonstrated the use of a diffusion tensor atlas as a prior distribution for the fibre orientation. Yap et al. [22] have similarly used a distribution of the maxima of the fibre orientation distributions (FOD) in different subjects, as a means of improving tractography of the average atlas. Finally, Yendiki et al. [23] imposed a shape prior on specific pathways connecting segmented end regions.

In this work, we extend the global tractography framework of Reisert et al. [18] with a prior on the local track orientation distribution (TOD) [5]. This prior is derived from 20 normal subjects and represented as an atlas in the basis of spherical harmonics (SH), that captures both the expected fibre directions and their support by the local neighbourhood. We expect this atlas to guide the global tractography towards a more targeted reconstruction, due to its higher angular contrast. In contrast to the current state of the art, our method finds the globally optimal tractogram without restrictions to specific pathways.

\section{Methods}

\subsection{Global Tractography in the SH Basis}

\subsubsection{Generative Model.}

The set of tracks we aim to reconstruct, i.e., the fibre model $\mathscr{M}$, is represented as a set of segments $\left\{\left(\mathbf{r}_{i}, \mathbf{n}_{i}\right)\right\}$, with position $\mathbf{r}_{i}$, direction $\mathbf{n}_{i}$, and fixed length $2 \ell$, and a set of connections between their endpoints. Ultimately, we wish to maximize the posterior probability of $\mathscr{M}$ given the data $D$, which, according to Bayes' rule and assuming an exponential model, equals

$$
P(\mathscr{M} \mid D) \propto P(D \mid \mathscr{M}) P(\mathscr{M})=e^{-E_{\text {ext }}(\mathscr{M}, D) / T} e^{-E_{\text {int }}(\mathscr{M}) / T} .
$$


As such, the problem becomes finding the global minimum of $E(\mathscr{M})=E_{\text {int }}(\mathscr{M})+$ $E_{\text {ext }}(\mathscr{M}, D)$. The internal energy $E_{\text {int }}$ promotes connectivity and smoothness of the reconstructed tracks, and is defined identically as in [18]. The external energy $E_{\text {ext }}$ measures the similarity to the data, defined as the mean squared error $\lambda_{\text {ext }} \| D(b, \mathbf{g})-$ $D^{\prime}(b, \mathbf{g}) \|^{2}$ between the measured data $D$ and the predicted data $D^{\prime}$ given the fibre model, for all $b$-values and gradient directions $\mathbf{g}$.

Given the model $\mathscr{M}$, we simulate the data $D^{\prime}$ by assuming that each segment has an equal contribution to the predicted signal $D^{\prime}$ in the form of a fibre response kernel $K_{b}(\theta)$, as used in spherical deconvolution techniques [4,19]. $K_{b}(\theta)$ is a spherical function depending only on the elevation, that models the expected diffusion signal for a single fibre direction along the $z$-axis. The data is then simulated by rotating this kernel along all segments, and integrating over all segments in a voxel $V(\mathbf{r})$, i.e., $D_{b}^{\prime}(\mathbf{u})=\sum_{i \in V(\mathbf{r})} K_{b}\left(\arccos \left(\mathbf{u} \cdot \mathbf{n}_{i}\right)\right)$. Cast into the real, symmetric SH basis [4], this becomes $D_{b}^{\prime}(\mathbf{u})=\sum_{i} K_{b} * \delta_{\mathbf{n}_{i}}(\mathbf{u})=K_{b} * \Psi(\mathbf{u})$ where $\delta_{\mathbf{n}_{i}}(\mathbf{u})$ is the SH Dirac delta function along direction $\mathbf{n}_{i}, *$ is the spherical convolution operator, and $\Psi(\mathbf{u})=$ $\sum_{i} \delta_{\mathbf{n}_{i}}(\mathbf{u})$ models the orientation distribution of the segments in that voxel. In addition, we introduce one or more isotropic kernels $c(b)$ that account for partial volume contamination of other tissue types and depend only on the $b$-value. In summary, the predicted DWI signal in every voxel equals

$$
D^{\prime}(b, \mathbf{g})=K_{b} * \Psi(\mathbf{g})+\sum_{j} f_{j} c_{j}(b),
$$

where $f_{j}$ are the respective isotropic fractions, estimated in each voxel as the leastsquares solution of the external energy, given the current segment configuration.

The intermediary representation $\Psi(\mathbf{u})$ of the fibre model is related to the recently introduced TOD [5] by convolution with an apodized point spread function (aPSF), defined as the sharpest non-negative function that can be represented in the SH basis of order $\ell_{\max }$. We denote the resulting TOD as $\tilde{\Psi}(\mathbf{u})=\operatorname{aPSF} * \Psi(\mathbf{u})$, a non-negative distribution that is more robust to $\mathrm{SH}$ aliasing effects. Note that for global tractography, the TOD closely resembles the FOD obtained from constrained spherical deconvolution [19], as the reconstructed track distribution is optimized for maximal correspondence to the data.

\subsubsection{Optimization.}

The optimization of (1) relies on the Metropolis-Hastings (MH) algorithm, a Markov Chain Monte Carlo technique, to obtain random samples from the posterior distribution [9]. As in simulated annealing methods, the temperature $T$ is gradually cooled down to increase the likelihood of sampling from the maximum of $P(\mathscr{M} \mid D)$ (or the minimum of $E(\mathscr{M})$ ). At each iteration, the MH sampler proposes a new state $\mathscr{M}^{\prime}$, obtained as a random perturbation of the current state $\mathscr{M}$, and evaluates the Green's ratio

$$
R=\min \left(1, \frac{e^{-E\left(\mathscr{M}^{\prime}\right) / T}}{e^{-E(\mathscr{M}) / T}} \frac{p^{\operatorname{prop}}\left(\mathscr{M} \mid \mathscr{M}^{\prime}\right)}{p^{\operatorname{prop}}\left(\mathscr{M}^{\prime} \mid \mathscr{M}\right)}\right)
$$


where $p^{\text {prop }}(y \mid x)$ is the transition probability from state $x$ to state $y$. The proposed state $\mathscr{M}^{\prime}$ is then accepted with probability $R$, or discarded otherwise. Transition proposals include birth/death of segments, random and optimal shifts, and creation/deletion of connections $[11,18]$.

\subsection{Atlas Prior}

Given an atlas $A$ of the local track orientation, the posterior probability of the fibre model equals $P(\mathscr{M} \mid D, A) \propto P(D \mid \mathscr{M}, A) P(\mathscr{M} \mid A) \propto P(D \mid \mathscr{M}) P(A \mid \mathscr{M}) P(\mathscr{M})$, using Bayes' rule and assuming the subject data is independent of the atlas. Assuming the usual exponential model, the new energy function becomes

$$
E(\mathscr{M})=E_{\text {ext }}(\mathscr{M}, D)+E_{\text {atlas }}(\mathscr{M}, A)+E_{\text {int }}(\mathscr{M}) .
$$

Hence, the atlas prior is a direct extension of the global tractography framework explained in the previous section, and can be optimized in the same way.

We define the atlas energy $E_{\text {atlas }}(\mathscr{M}, A)$ as the $L_{2}$-distance between the reconstructed TOD $\tilde{\Psi}(\mathbf{u})$ and the atlas TOD $\tilde{\Psi}_{a}(\mathbf{u})$, which, according to Parseval's theorem, equals the sum of squared differences between the corresponding SH coefficients. In the spirit of [5], we propose to use a minimum track length threshold for the atlas TOD, which emphasizes the neighbourhood support of the local track orientation. Note that by imposing such length threshold, the distinction between the TOD, which is a direct representation of any tractogram, and the FOD, which relates to the data, becomes important.

\section{Experiments and Results}

\subsection{Data}

Data of 25 neurologically healthy subjects between ages 22 and 35 years old were provided by the WU-Minn Human Connectome Project (HCP) Q3 data release [20]. The diffusion data consists of $3 \times 90$ gradient directions at $b$-values 1000, 2000, and $3000 \mathrm{~s} / \mathrm{mm}^{2}$ and 18 non-diffusion weighted images $(b=0)$, at an isotropic voxel size of $1.25 \mathrm{~mm}$, and was corrected for motion and EPI distortions as described in [8]. In each subject, the white matter (WM) response function was estimated in a fractional anisotropy (FA $\geq 0.75)$ mask using standard techniques for all shells [19]. Isotropic grey matter (GM) and cerebrospinal fluid (CSF) kernels were estimated as the average signal in manually delineated regions. Finally, the kernels of all subjects were averaged to obtain one mean WM, GM and CSF kernel. 


\subsection{Atlas Construction}

The atlas was constructed out of 20 randomly selected subjects, using our global tractography method without atlas prior in each of them individually. The segment length was set to $2 \mathrm{~mm}$, the maximal order of the $\mathrm{SH}$ basis to $\ell_{\max }=10$, and the weight of each segment to 0.1 , such that on average 10 segments per white matter voxel are reconstructed. We ran the $\mathrm{MH}$ sampler for $10^{9}$ iterations, which took around 10 hours on a standard desktop computer.

The resulting fibre segments were normalized to MNI space, using the nonlinear warps provided by the HCP and originally obtained from FSL FNIRT [8]. The centre point of each segment is transformed to atlas space and its direction is reoriented according to the Jacobian of the local deformation field. Next, we imposed a minimum track length threshold, such that only tracks consisting of at least 10 segments remained. These segments were subsequently transformed and reoriented to the space of the 5 remaining subjects for testing. Finally, the set of transformed and filtered segments of all subjects was converted to a TOD representation using aPSFs as described in Section 2.1. Conceptually, this is identical to existing methods for FOD/TOD reorientation and atlas construction [2,17], except that those methods first fit a weighted sum of uniformly oriented aPSFs to the data in every voxel. The segment representation, used in global tractography, allows to avoid this fitting step.

\subsection{Evaluation}

The effect of the proposed atlas prior was evaluated in the 5 remaining subjects, using identical parameter settings as for the initial global tractography. First of all, the mean track length, reported in Table 1, is exponentially distributed and increases significantly with the atlas prior (F-test, $p$-value $10^{-6}$ ) by about $25 \%$. Simultaneously, the imposed atlas prior reduces the number of tracks by approximately $50 \%$. These observations indicate that while the overall density decreases, mostly the number of short, incomplete or interrupted tracks is reduced by the atlas prior.

Table 1 Mean track length and the total number of reconstructed tracks for the 5 test subjects.

\begin{tabular}{lccccc}
\hline & \multicolumn{2}{c}{ uniform prior } & & \multicolumn{2}{c}{ atlas prior } \\
\cline { 2 - 3 } \cline { 5 - 5 } Subject & avg. length $(\mathrm{mm})$ & no. tracks & & avg. length $(\mathrm{mm})$ & no. tracks \\
\hline 1 & 16.05 & 281403 & & 19.27 & 131196 \\
2 & 17.04 & 211759 & & 21.93 & 112591 \\
3 & 16.16 & 249838 & & 20.48 & 119286 \\
4 & 16.79 & 199610 & & 21.93 & 109324 \\
5 & 16.98 & 221455 & & 21.57 & 116499 \\
\hline
\end{tabular}


Figure 1 shows a cross-section of the reconstructed tractogram of test subject 1 , and illustrates that the density decrease with the atlas prior is mainly located in the distal gyri. All major WM bundles are present in the reconstruction, and the crossing of the corpus callosum, the corona radiata, and the superior longitudinal fasciculus, sometimes challenging for tractography, is successfully recovered in both global tractography reconstructions, with and without the atlas prior. However, a close-up of the TOD in this region, as shown in Fig. 2, illustrates that the main fibre directions are more consistent across neighbouring voxels when using the atlas prior. Similar results were observed in the remaining test subjects (not shown).

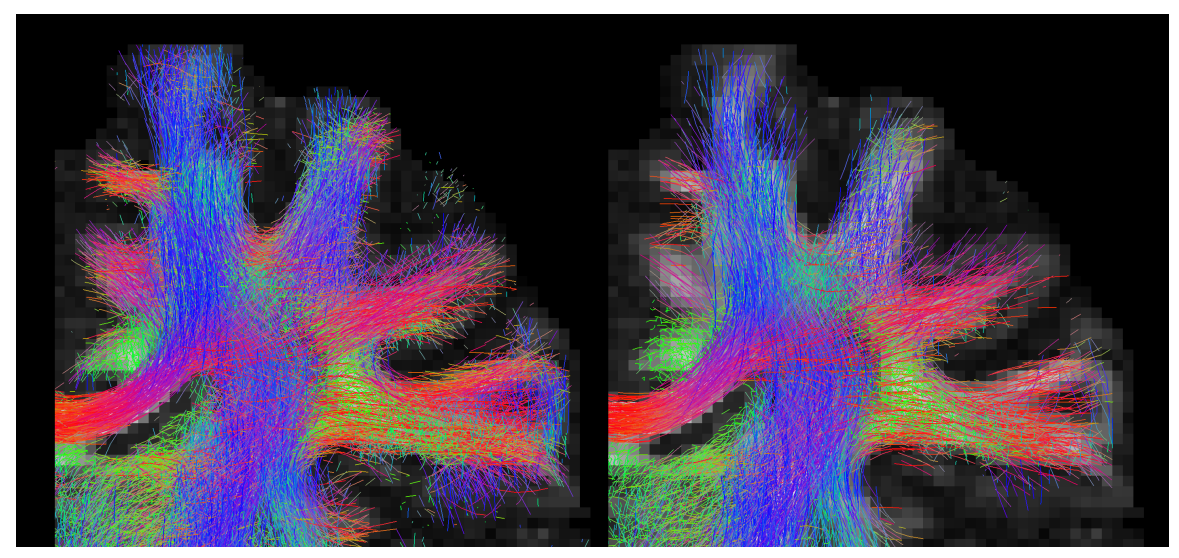

Fig. 1 Coronal slab $(5 \mathrm{~mm})$ of the reconstructed tractogram in the cerebrum, overlaid on a fractional anisotropy map, without atlas prior (left), and with atlas prior (right).
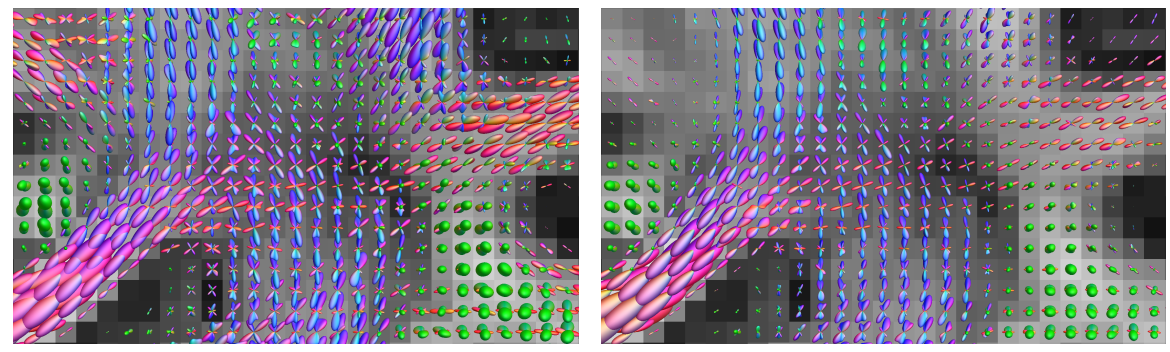

Fig. 2 Track orientation distribution (TOD) in the centrum semiovale, obtained without atlas prior (left), and with atlas prior (right). With atlas prior, the track orientations are more consistent across neighbouring voxels.

Finally, we segmented the left cingulate tract in both reconstructions based on a WM parcellation obtained with FreeSurfer [7] following the protocol described 
in [21]. As shown in Fig. 3, the atlas prior strongly reduces the amount of spurious tracks that run from the cingulum into the corpus callosum. Hence, incorporating the atlas prior improves the specificity of the track reconstruction.
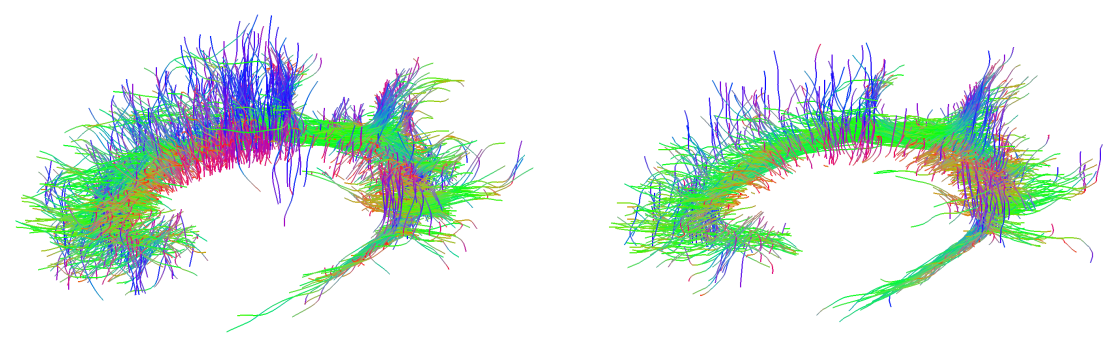

Fig. 3 Sagittal view of the left cingulate tract, segmented from the global fibre reconstruction without atlas prior (left), and with atlas prior (right). Notice that with the prior, the number of false positive connections to the corpus callosum is reduced.

\section{Discussion}

At a conceptual level, there are many analogies between tractography and segmentation. Both aim to delineate structures of interest, both rely on neighbourhood information for doing so, and both aim to reconstruct shapes with remarkable similarity across subjects. As such, we can expect that tractography, like segmentation, can benefit from inter-subject information in the shape of an atlas that captures the expected anatomy.

The global tractography framework is well suited for the inclusion of such atlas prior, as it aims to reconstruct the optimal fibre configuration in the whole image volume. Indeed, we have shown that a prior of the local track orientation can be elegantly included as an additional energy term in the optimization. Selecting the weight of this atlas energy allows to set the reliance on the atlas relative to the subject data, whereas the internal energy ensures spatial continuity.

While the atlas contains only local information, the imposed minimum track length ensures "track-like local support" in the neighbouring voxels [5], which can be a powerful prior for guiding the fibre reconstruction. This is particularly evidenced by our results in Figs. 2 and 3, in which we observed more consistent track orientations and fewer spurious connections. An alternative application of the presented method could be to use a high-resolution atlas, built from HCP data, in conjunction with data of lower spatial and angular resolution. As such, the reconstruction of clinical data with fast acquisition schemes may be improved. 
Nevertheless, our proposed atlas-guided tractography method also suffers from some of the limitations of atlas-based segmentation methods. Foremost, the required atlas-to-subject registration can introduce misalignment artefacts that make for a fuzzy prior at best, and a plain wrong one at worst. We noticed the effect of such artefacts in some of the distal gyri, where a "null prior" was mapped onto the gyrus, disabling any track reconstruction in that area. Secondly, the robustness of the current setup to pathology is in question, as registration in the presence of (tumorous) lesions is still an open issue.

\section{Conclusion}

This work is, to the best of our knowledge, the first to include a track orientation prior in a global tractography framework. Our method has a beneficial effect on the reconstructed tractogram, although its success depends on the quality of the registration. Future work can focus on alternative atlas measures and multi-atlas approaches, which may perform better in this regard.

Acknowledgements D. Christiaens is supported by a Ph.D. grant of the Agency for Innovation by Science and Technology (IWT). Data were provided by the Human Connectome Project, WU-Minn Consortium (Principal Investigators: David Van Essen and Kamil Ugurbil; 1U54MH091657) funded by the 16 NIH Institutes and Centers that support the NIH Blueprint for Neuroscience Research; and by the McDonnell Center for Systems Neuroscience at Washington University.

\section{References}

1. Cabezas, M., Oliver, A., Lladó, X., Freixenet, J., Bach Cuadra, M.: A review of atlas-based segmentation for magnetic resonance brain images. Computer methods and programs in biomedicine 104(3), e158-e177 (2011)

2. Christiaens, D., Dhollander, T., Maes, F., Sunaert, S., Suetens, P.: Groupwise deformable registration of fiber track sets using track orientation distributions. In: T. Schultz, G. NedjatiGilani, A. Venkataraman, L. O’Donnell, E. Panagiotaki (eds.) Computational Diffusion MRI and Brain Connectivity, Mathematics and Visualization, pp. 151-161. Springer International Publishing (2014)

3. Cook, P.A., Zhang, H., Awate, S.P., Gee, J.C.: Atlas-guided probabilistic diffusion-tensor fiber tractography. In: 5th International Symposium on Biomedical Imaging: From Nano to Macro ISBI 2008, pp. 951-954. IEEE (2008)

4. Descoteaux, M., Deriche, R., Knosche, T., Anwander, A.: Deterministic and probabilistic tractography based on complex fibre orientation distributions. IEEE Transactions on Medical Imaging 28(2), 269-286 (2009)

5. Dhollander, T., Emsell, L., Van Hecke, W., Maes, F., Sunaert, S., Suetens, P.: Track orientation density imaging (TODI) and track orientation distribution (TOD) based tractography. NeuroImage (in press)

6. Fillard, P., Poupon, C., Mangin, J.F.: A novel global tractography algorithm based on an adaptive spin glass model. In: G.Z. Yang, D. Hawkes, D. Rueckert, A. Noble, C. Taylor (eds.) 
Medical Image Computing and Computer-Assisted Intervention - MICCAI 2009, LNCS, vol. 5761, pp. 927-934. Springer Berlin Heidelberg (2009)

7. Fischl, B.: Freesurfer. NeuroImage 62(2), 774-781 (2012)

8. Glasser, M.F., Sotiropoulos, S.N., Wilson, J.A., Coalson, T.S., Fischl, B., Andersson, J.L., Xu, J., Jbabdi, S., Webster, M., Polimeni, J.R., Van Essen, D.C., Jenkinson, M.: The minimal preprocessing pipelines for the human connectome project. NeuroImage (2013)

9. Hastings, W.K.: Monte Carlo sampling methods using Markov chains and their applications. Biometrika 57(1), 97-109 (1970)

10. Jbabdi, S., Johansen-Berg, H.: Tractography: where do we go from here? Brain Connectivity 1(3), 169-183 (2011)

11. Kreher, B., Mader, I., Kiselev, V.: Gibbs tracking: a novel approach for the reconstruction of neuronal pathways. Magnetic Resonance in Medicine 60(4), 953-963 (2008)

12. Mangin, J.F., Fillard, P., Cointepas, Y., Le Bihan, D., Frouin, V., Poupon, C.: Toward global tractography. NeuroImage 80, 290-296 (2013)

13. Mangin, J.F., Poupon, C., Cointepas, Y., Riviere, D., Papadopoulos-Orfanos, D., Clark, C., Régis, J., Le Bihan, D.: A framework based on spin glass models for the inference of anatomical connectivity from diffusion-weighted MR data-a technical review. NMR in Biomedicine 15(7-8), 481-492 (2002)

14. Mori, S., van Zijl, P.: Fiber tracking: principles and strategies-a technical review. NMR in Biomedicine 15(7-8), 468-480 (2002)

15. O'Donnell, L.J., Golby, A.J., Westin, C.F.: Fiber clustering versus the parcellation-based connectome. NeuroImage 80, 283-289 (2013)

16. Parker, G.J.: Probabilistic fiber tracking. In: D.K. Jones (ed.) Diffusion MRI: Theory, methods, and applications, pp. 396-408. Oxford University Press (2010)

17. Raffelt, D., Tournier, J., Crozier, S., Connelly, A., Salvado, O.: Reorientation of fiber orientation distributions using apodized point spread functions. Magnetic Resonance in Medicine 67(3), 844-855 (2012)

18. Reisert, M., Mader, I., Anastasopoulos, C., Weigel, M., Schnell, S., Kiselev, V.: Global fiber reconstruction becomes practical. NeuroImage 54(2), 955-962 (2011)

19. Tournier, J., Calamante, F., Connelly, A.: Robust determination of the fibre orientation distribution in diffusion MRI: non-negativity constrained super-resolved spherical deconvolution. NeuroImage 35(4), 1459-1472 (2007)

20. Van Essen, D.C., Smith, S.M., Barch, D.M., Behrens, T.E., Yacoub, E., Ugurbil, K.: The WU-Minn human connectome project: an overview. NeuroImage 80, 62-79 (2013)

21. Wassermann, D., Makris, N., Rathi, Y., Shenton, M., Kikinis, R., Kubicki, M., Westin, C.F.: On describing human white matter anatomy: The white matter query language. In: K. Mori, I. Sakuma, Y. Sato, C. Barillot, N. Navab (eds.) Medical Image Computing and ComputerAssisted Intervention - MICCAI 2013, Lecture Notes in Computer Science, vol. 8149, pp. 647-654. Springer Berlin Heidelberg (2013)

22. Yap, P.T., Gilmore, J.H., Lin, W., Shen, D.: PopTract: population-based tractography. IEEE Transactions on Medical Imaging 30(10), 1829-1840 (2011)

23. Yendiki, A., Panneck, P., Srinivasan, P., Stevens, A., Zöllei, L., Augustinack, J., Wang, R., Salat, D., Ehrlich, S., Behrens, T., Jbabdi, S., Gollub, R., Fischl, B.: Automated probabilistic reconstruction of white-matter pathways in health and disease using an atlas of the underlying anatomy. Frontiers in neuroinformatics 5 (2011) 